\title{
A PENALTY ALGORITHM FOR THE SPECTRAL ELEMENT DISCRETIZATION OF THE STOKES PROBLEM*
}

\author{
Christine Bernardi $^{1}$, Adel Blouza ${ }^{2}$, Nejmeddine Chorfi $^{3}$ and Nizar Kharrat ${ }^{4}$
}

\begin{abstract}
The penalty method when applied to the Stokes problem provides a very efficient algorithm for solving any discretization of this problem since it gives rise to a system of two equations where the unknowns are uncoupled. For a spectral or spectral element discretization of the Stokes problem, we prove a posteriori estimates that allow us to optimize the penalty parameter as a function of the discretization parameter. Numerical experiments confirm the interest of this technique.
\end{abstract}

Mathematics Subject Classification. 76D07, 76M22.

Received June 18, 2008. Revised September 18, 2009.

Published online August 2, 2010.

\section{INTRODUCTION}

The Stokes problem models the laminar flow of a viscous incompressible fluid in a two- or three-dimensional domain. Its unknowns are the velocity and the pressure of the fluid. Any discretization of this problem by Galerkin type methods results into a linear system of two coupled equations. A large number of algorithms exist to uncouple the two unknowns, see $[14,15]$, and the references therein. In this work, we are interested in the penalty method for spectral element discretizations.

Indeed, the penalty method, as described in [14], Chapter I, Section 4.3, in an abstract framework, has been extensively used in the case of finite element discretizations, see [2,3] and [16] for the first a priori error analysis and numerical experiments, and [11-13] for complementary results. However, up to our knowledge, this method has not so far been considered in the context of spectral and spectral element discretizations. The main reason can be expressed as follows: The high accuracy of spectral methods and the convergence of order 1 with respect to the penalty parameter would lead to choose a very small penalty parameter in order to equilibrate the two types of errors and, as a consequence, the condition number of the matrix that must be inverted would be very high. Nevertheless, we think that the penalty method is very interesting in the framework of spectral methods

\footnotetext{
Keywords and phrases. Stokes problem, spectral elements, penalty algorithm.

* N. Chorfi was supported by King Saud University, D.S.F.P. Program.

${ }^{1}$ Laboratoire Jacques-Louis Lions, C.N.R.S. \& Université Pierre et Marie Curie, B.C. 187, 4 place Jussieu, 75252 Paris Cedex 05, France. bernardi@ann.jussieu.fr

${ }^{2}$ Laboratoire de Mathématiques Raphaël Salem (U.M.R. 6085 C.N.R.S.), Université de Rouen, avenue de l'Université, B.P. 12, 76801 Saint-Étienne-du-Rouvray, France. Adel.Blouza@univ-rouen.fr

3 Department of Mathematics, College of Sciences, King Saud University, Riyadh 11451, Saudi Arabia.

nejmeddine.chorfi@fst.rnu.tn

4 Faculté des Sciences de Bizerte, ENIT-LAMSIN, B.P. 37, 1002 Tunis Le Belvédère, Tunisia. nizar.kharrat@enit.rnu.tn
} 
for the next two reasons:

(i) It is well-known [16] in the case of finite element discretizations that the addition of a penalty term stabilizes the discrete problem when the constant on the inf-sup condition for the pressure is not independent of the discretization parameter, which is the case for most spectral methods, see [6], Sections 24-26;

(ii) Still in the case of finite elements, it has been recently proved in [9] that the construction of appropriate error indicators leads to optimizing the choice of the penalty parameter for a fixed discretization, more precisely to choose this parameter such that the penalty error and the discretization error are of the same order. The main interest of this optimization is a high reduction of the computation cost for solving the discrete problem.

So, this paper is aimed to the a posteriori analysis of the penalized spectral element discretization of the Stokes problem. Numerical experiments confirm the interest of such an algorithm and also allow us to compare the cases of discretizations with optimal or non optimal inf-sup constants.

An outline of the paper is as follows.

- In Section 2, we describe the continuous, penalized and discrete Stokes problems and recall their main properties.

- Section 3 is devoted to the a posteriori analysis of the penalized discrete problem.

- In Section 4, we describe the strategy that is used in order to optimize the choice of the penalty parameter and present some numerical experiments concerning the penalty spectral element method.

\section{The COntinuous, PEnAlized AND DisCRETE PROBlems}

Let $\Omega$ be a bounded connected open set in $\mathbb{R}^{d}, d=2$ or 3 , with a Lipschitz-continuous boundary $\partial \Omega$. The Stokes problem in this domain reads

$$
\left\{\begin{array}{cl}
-\nu \Delta \boldsymbol{u}+\operatorname{grad} p=\boldsymbol{f} & \text { in } \Omega \\
\operatorname{div} \boldsymbol{u}=0 & \text { in } \Omega \\
\boldsymbol{u}=\mathbf{0} & \text { on } \partial \Omega
\end{array}\right.
$$

where the unknowns are the velocity $\boldsymbol{u}$ and the pressure $p$. The datum $\boldsymbol{f}$ represents a density of body forces and the viscosity $\nu$ is a positive constant.

Remark 2.1. We have chosen to work with homogeneous boundary conditions on the velocity for simplicity. Indeed, extending our results to the nonhomogeneous conditions $\boldsymbol{u}=\mathbf{g}$ on $\partial \Omega$ only involves some further technical difficulties which can be solved via standard arguments. However it seems that the penalty algorithm has not been applied so far to the case of mixed boundary conditions whatever the discretization is.

We use the standard notation for the Sobolev spaces $H^{s}(\Omega), s \in \mathbb{R}$, and $H_{0}^{s}(\Omega), s \geq 0$, provided with the corresponding norms. We denote by $L_{0}^{2}(\Omega)$ the space of functions in $L^{2}(\Omega)$ with a null integral on $\Omega$. Thus, for any source term $\boldsymbol{f}$ in $H^{-1}(\Omega)^{d}$, problem $(2.1)$ admits the equivalent variational formulation:

Find $(\boldsymbol{u}, p)$ in $H_{0}^{1}(\Omega)^{d} \times L_{0}^{2}(\Omega)$ such that

$$
\begin{aligned}
& \forall \boldsymbol{v} \in H_{0}^{1}(\Omega)^{d}, \quad a(\boldsymbol{u}, \boldsymbol{v})+b(\boldsymbol{v}, p)=\langle\boldsymbol{f}, \boldsymbol{v}\rangle, \\
& \forall q \in L_{0}^{2}(\Omega), \quad b(\boldsymbol{u}, q)=0,
\end{aligned}
$$

where the bilinear forms $a(\cdot, \cdot)$ and $b(\cdot, \cdot)$ are defined by

$$
\begin{gathered}
a(\boldsymbol{u}, \boldsymbol{v})=\nu \int_{\Omega} \operatorname{grad} \boldsymbol{u}: \operatorname{grad} \boldsymbol{v} \mathrm{d} \boldsymbol{x}, \\
b(\boldsymbol{v}, q)=-\int_{\Omega}(\operatorname{div} \boldsymbol{v})(\boldsymbol{x}) q(\boldsymbol{x}) \mathrm{d} \boldsymbol{x}
\end{gathered}
$$

while $\langle\cdot, \cdot\rangle$ stands for the duality pairing between $H^{-1}(\Omega)$ and $H_{0}^{1}(\Omega)$. 
We recall the following properties, see [14], Chapter I, for instance:

(i) There exists a constant $\alpha>0$ such that the following ellipticity property holds

$$
\forall \boldsymbol{v} \in H_{0}^{1}(\Omega)^{d}, \quad a(\boldsymbol{v}, \boldsymbol{v}) \geq \alpha\|\boldsymbol{v}\|_{H^{1}(\Omega)^{d}}^{2} ;
$$

(ii) There exists a constant $\beta>0$ such that the following inf-sup condition holds

$$
\forall q \in L_{0}^{2}(\Omega), \quad \sup _{\boldsymbol{v} \in H_{0}^{1}(\Omega)^{d}} \frac{b(\boldsymbol{v}, q)}{\|\boldsymbol{v}\|_{H^{1}(\Omega)^{d}}} \geq \beta\|q\|_{L^{2}(\Omega)} .
$$

Thus, it is readily checked ([14], Chap. I, Cor. 4.1) that problem (2.2) admits a unique solution $(\boldsymbol{u}, p)$ in $H_{0}^{1}(\Omega)^{d} \times L_{0}^{2}(\Omega)$ which moreover satisfies

$$
\|\boldsymbol{u}\|_{H^{1}(\Omega)^{d}}+\|p\|_{L^{2}(\Omega)} \leq c\|\boldsymbol{f}\|_{H^{-1}(\Omega)^{d}} .
$$

Let now $\varepsilon$ be a penalty parameter, $0<\varepsilon \leq 1$. We consider the penalized problem

Find $\left(\boldsymbol{u}^{\varepsilon}, p^{\varepsilon}\right)$ in $H_{0}^{1}(\Omega)^{d} \times L_{0}^{2}(\Omega)$ such that

$$
\begin{aligned}
& \forall \boldsymbol{v} \in H_{0}^{1}(\Omega)^{d}, \quad a\left(\boldsymbol{u}^{\varepsilon}, \boldsymbol{v}\right)+b\left(\boldsymbol{v}, p^{\varepsilon}\right)=\langle\boldsymbol{f}, \boldsymbol{v}\rangle, \\
& \forall q \in L_{0}^{2}(\Omega), \quad b\left(\boldsymbol{u}^{\varepsilon}, q\right)=\varepsilon \int_{\Omega} p^{\varepsilon}(\boldsymbol{x}) q(\boldsymbol{x}) \mathrm{d} \boldsymbol{x} .
\end{aligned}
$$

We recall from [14], Chapter I, Theorem 4.3, the following result.

Proposition 2.2. For any source term $\boldsymbol{f}$ in $H^{-1}(\Omega)^{d}$, problem (2.7) has a unique solution $\left(\boldsymbol{u}^{\varepsilon}, p^{\varepsilon}\right)$ in $H_{0}^{1}(\Omega)^{d} \times$ $L_{0}^{2}(\Omega)$. Moreover the following estimate holds between this solution and the solution $(\boldsymbol{u}, p)$ of problem $(2.2)$

$$
\left\|\boldsymbol{u}-\boldsymbol{u}^{\varepsilon}\right\|_{H^{1}(\Omega)^{d}}+\left\|p-p^{\varepsilon}\right\|_{L^{2}(\Omega)} \leq c \varepsilon\|\boldsymbol{f}\|_{H^{-1}(\Omega)^{d}} .
$$

To describe the discrete problem, we introduce a partition of the domain $\Omega$ without overlap:

$$
\bar{\Omega}=\cup_{k=1}^{K} \bar{\Omega}_{k} \quad \text { and } \quad \Omega_{k} \cap \Omega_{k^{\prime}}=\emptyset, \quad 1 \leq k<k^{\prime} \leq K,
$$

where each $\Omega_{k}$ is a rectangle in dimension $d=2$, a rectangular parallelepiped in dimension $d=3$ (note that the method can easily be extended to curved rectangles or rectangular parallelepipeds, see [10], Sect. VI.1). We make the further assumption that the intersection of two different $\bar{\Omega}_{k}$, if not empty, is either a vertex or a whole edge or a whole face of the two subdomains. We take without restriction the edges of the $\Omega_{k}$ parallel to the coordinate axes.

For each nonnegative real number $s$, let $\mathbb{P}_{s}\left(\Omega_{k}\right)$ be the space of restrictions to $\Omega_{k}$ of polynomials with $d$ variables and degree smaller than the integer part of $s$ with respect to each variable. Let now $N$ be an integer, $N \geq 2$. We introduce the discrete spaces

$$
\mathbb{X}_{N}=\left\{\boldsymbol{v}_{N} \in H_{0}^{1}(\Omega)^{d} ; \boldsymbol{v}_{N \mid \Omega_{k}} \in \mathbb{P}_{N}\left(\Omega_{k}\right)^{d}, 1 \leq k \leq K\right\},
$$

and, for a fixed real number $\lambda, 0<\lambda \leq 1$,

$$
\mathbb{M}_{N}=\left\{q_{N} \in L_{0}^{2}(\Omega) ; q_{N \mid \Omega_{k}} \in \mathbb{P}_{N-2}\left(\Omega_{k}\right) \cap \mathbb{P}_{\lambda N}\left(\Omega_{k}\right), 1 \leq k \leq K\right\} .
$$

The reason for this choice is that, even for $\lambda=1$, the space $\mathbb{M}_{N}$ does not contain spurious modes. But the constant of the discrete inf-sup condition on the form $b(\cdot, \cdot)$ is independent of $N$ only for $\lambda<1$, see [6], Sections 24-26, and [7], Proposition 3.1. 
We recall the standard properties of the Gauss-Lobatto-Legendre formula on $]-1,1\left[\right.$ : With $\xi_{0}=-1$ and $\xi_{N}=1$, there exist a unique set of nodes $\xi_{j}, 1 \leq j \leq N-1$, in ]-1, 1 [ and a unique set of weights $\rho_{j}, 0 \leq j \leq N$, such that

$$
\forall \Phi \in \mathbb{P}_{2 N-1}(-1,1), \quad \int_{-1}^{1} \Phi(\zeta) \mathrm{d} \zeta=\sum_{j=0}^{N} \Phi\left(\xi_{j}\right) \rho_{j}
$$

Moreover, the $\rho_{j}$ are positive and the following property holds, see [6], Formula (13.20):

$$
\forall \varphi_{N} \in \mathbb{P}_{N}(-1,1), \quad\left\|\varphi_{N}\right\|_{L^{2}(-1,1)}^{2} \leq \sum_{j=0}^{N} \varphi_{N}^{2}\left(\xi_{j}\right) \rho_{j} \leq 3\left\|\varphi_{N}\right\|_{L^{2}(-1,1)}^{2}
$$

Denoting by $F_{k}$ one of the mappings which send the square or cube $]-1,1\left[{ }^{d}\right.$ onto $\Omega_{k}$, we define the discrete product, for any functions $u$ and $v$ continuous on $\bar{\Omega}$,

$$
(u, v)_{N}= \begin{cases}\sum_{k=1}^{K} \frac{\operatorname{meas}\left(\Omega_{k}\right)}{4} \sum_{i=0}^{N} \sum_{j=0}^{N} u \circ F_{k}\left(\xi_{i}, \xi_{j}\right) v \circ F_{k}\left(\xi_{i}, \xi_{j}\right) \rho_{i} \rho_{j} & \text { if } d=2, \\ \sum_{k=1}^{K} \frac{\operatorname{meas}\left(\Omega_{k}\right)}{8} \sum_{i=0}^{N} \sum_{j=0}^{N} \sum_{p=0}^{N} u \circ F_{k}\left(\xi_{i}, \xi_{j}, \xi_{p}\right) v \circ F_{k}\left(\xi_{i}, \xi_{j}, \xi_{p}\right) \rho_{i} \rho_{j} \rho_{p} & \text { if } d=3 .\end{cases}
$$

We also introduce the associated Lagrange interpolation operator $\mathcal{I}_{N}$ : For any continuous function $g$ on $\bar{\Omega}$, $\mathcal{I}_{N} g_{\mid \Omega_{k}}$ belongs to $\mathbb{P}_{N}\left(\Omega_{k}\right)$ and is equal to $g$ at all nodes $F_{k}\left(\xi_{i}, \xi_{j}\right), 0 \leq i, j \leq N$, in dimension $d=2$, at all nodes $F_{k}\left(\xi_{i}, \xi_{j}, \xi_{p}\right), 0 \leq i, j, p \leq N$, in dimension $d=3$.

We are now in a position to write the discrete problem: For any source term $f$ continuous on $\bar{\Omega}$,

Find $\left(\boldsymbol{u}_{N}^{\varepsilon}, p_{N}^{\varepsilon}\right)$ in $\mathbb{X}_{N} \times \mathbb{M}_{N}$ such that

$$
\begin{aligned}
& \forall \boldsymbol{v}_{N} \in \mathbb{X}_{N}, \quad a_{N}\left(\boldsymbol{u}_{N}^{\varepsilon}, \boldsymbol{v}_{N}\right)+b_{N}\left(\boldsymbol{v}_{N}, p_{N}^{\varepsilon}\right)=\left(\boldsymbol{f}, \boldsymbol{v}_{N}\right)_{N}, \\
& \forall q_{N} \in \mathbb{M}_{N}, \quad b_{N}\left(\boldsymbol{u}_{N}^{\varepsilon}, q_{N}\right)=\varepsilon\left(p_{N}^{\varepsilon}, q_{N}\right)_{N},
\end{aligned}
$$

where the bilinear forms $a_{N}(\cdot, \cdot)$ and $b_{N}(\cdot, \cdot)$ are defined by

$$
a_{N}\left(\boldsymbol{u}_{N}, \boldsymbol{v}_{N}\right)=\nu\left(\operatorname{grad} \boldsymbol{u}_{N}, \operatorname{grad} \boldsymbol{v}_{N}\right)_{N}, \quad b_{N}\left(\boldsymbol{v}_{N}, q_{N}\right)=-\left(\operatorname{div} \boldsymbol{v}_{N}, q_{N}\right)_{N} .
$$

Note that, thanks to the exactness property $(2.11)$ and the choice of $\mathbb{M}_{N}, b_{N}(\cdot, \cdot)$ can be replaced by $b(\cdot, \cdot)$ in this problem; similarly, in the second line of this problem, the discrete product $(\cdot, \cdot)_{N}$ can be replaced by the scalar product of $L^{2}(\Omega)$.

We now explain the main advantage of this discrete problem with respect to the standard discrete Stokes problem. Let $\Pi_{N}$ denote the orthogonal projection operator from $L_{0}^{2}(\Omega)$ onto $\mathbb{M}_{N}$. Then problem (2.13) is fully equivalent (see [14], Chap. I, Sect. 4.3) to the system

$$
\forall \boldsymbol{v}_{N} \in \mathbb{X}_{N}, \quad a_{N}\left(\boldsymbol{u}_{N}^{\varepsilon}, \boldsymbol{v}_{N}\right)+\varepsilon^{-1}\left(\Pi_{N}\left(\operatorname{div} \boldsymbol{u}_{N}^{\varepsilon}\right), \Pi_{N}\left(\operatorname{div} \boldsymbol{v}_{N}\right)\right)_{N}=\left(\boldsymbol{f}, \boldsymbol{v}_{N}\right)_{N}
$$

and

$$
p_{N}^{\varepsilon}=-\varepsilon^{-1} \Pi_{N}\left(\operatorname{div} \boldsymbol{u}_{N}^{\varepsilon}\right)
$$

The only unknown of equation (2.15) is the discrete velocity $\boldsymbol{u}_{N}^{\varepsilon}$ and equation (2.16) provides an explicit formula for the discrete pressure $p_{N}^{\varepsilon}$. Thus solving problem (2.13) is not at all expensive.

Proposition 2.3. For any source term $\boldsymbol{f}$ continuous on $\bar{\Omega}$, problem (2.13) has a unique solution $\left(\boldsymbol{u}_{N}^{\varepsilon}, p_{N}^{\varepsilon}\right)$.

Proof. The continuity of the bilinear forms involved in (2.13) follows from standard arguments, see [10], Chapter V, Proposition 1.1. The ellipticity of the form $a_{N}(\cdot, \cdot)$ (with an ellipticity constant independent of $N$ ) follows from (2.12) and a Poincaré-Friedrichs inequality. Next, when setting

$$
\tilde{a}_{N}\left(\boldsymbol{u}_{N}, \boldsymbol{v}_{N}\right)=a_{N}\left(\boldsymbol{u}_{N}, \boldsymbol{v}_{N}\right)+\varepsilon^{-1}\left(\Pi_{N}\left(\operatorname{div} \boldsymbol{u}_{N}\right), \Pi_{N}\left(\operatorname{div} \boldsymbol{v}_{N}\right)\right)_{N}
$$


we observe that $\tilde{a}_{N}\left(\boldsymbol{v}_{N}, \boldsymbol{v}_{N}\right) \geq a_{N}\left(\boldsymbol{v}_{N}, \boldsymbol{v}_{N}\right)$, whence the ellipticity of $\tilde{a}_{N}(\cdot, \cdot)$. Thus, problem (2.15) has a unique solution in $\mathbb{X}_{N}$. Thus, the pair $\left(\boldsymbol{u}_{N}^{\varepsilon}, p_{N}^{\varepsilon}\right)$, with $p_{N}^{\varepsilon}$ given by (2.16), is the unique solution to problem (2.13).

Moreover, we recall from [1], Proposition 5.1, the following inf-sup condition:

$$
\forall q_{N} \in \mathbb{M}_{N}, \quad \sup _{\boldsymbol{v}_{N} \in \mathbb{X}_{N}} \frac{b_{N}\left(\boldsymbol{v}_{N}, q_{N}\right)}{\left\|\boldsymbol{v}_{N}\right\|_{H^{1}(\Omega)^{d}}} \geq \beta_{N}(\lambda)\left\|q_{N}\right\|_{L^{2}(\Omega)},
$$

with

$$
\beta_{N}(\lambda)= \begin{cases}\beta_{1} N^{-\frac{d-1}{2}} & \text { if } \lambda=1, \\ \beta_{2} & \text { if } \lambda<1,\end{cases}
$$

where $\beta_{1}$ and $\beta_{2}$ are positive constants independent of $N$. More precisely, it follows from [7], Section 3, that:

(i) The constant $\beta_{1}$ is equal to a constant $c_{0}$ that can be explicitly computed times the constant $\beta$ in $(2.5)$ times $2^{\frac{d-1}{2}}$

(ii) The constant $\beta_{2}$ is equal to the same constant $c_{0}$ as previously times $\beta(1-\lambda)^{\frac{d-1}{2}}$.

We now establish the a priori error estimate.

Proposition 2.4. Assume that the source term $\boldsymbol{f}$ belongs to $H^{\sigma}(\Omega)^{d}, \sigma>\frac{d}{2}$, and that the solutions (u,p) to problem (2.2) and $\left(\boldsymbol{u}^{\varepsilon}, p^{\varepsilon}\right)$ to problem (2.7) belongs to $H^{s+1}(\Omega)^{d} \times H^{s}(\Omega), s \geq 0$. There exists a constant c only depending on the corresponding norms of these source term and solutions such that the following error estimate holds between the solutions $(\boldsymbol{u}, p)$ to problem $(2.2)$ and $\left(\boldsymbol{u}_{N}^{\varepsilon}, p_{N}^{\varepsilon}\right)$ to problem $(2.13)$

$$
\left\|\boldsymbol{u}-\boldsymbol{u}_{N}^{\varepsilon}\right\|_{H^{1}(\Omega)^{d}}+\mu\left\|p-p_{N}^{\varepsilon}\right\|_{L^{2}(\Omega)} \leq c\left(\varepsilon+\left(1+\mu^{-1}\right) N^{-s}+N^{-\sigma}\right),
$$

with $\mu=\max \left\{\beta_{N}(\lambda), \varepsilon\right\}$.

Proof. We proceed in two steps.

(1) Estimate (2.20) with $\mu=\beta_{N}(\lambda)$ is derived firstly by bounding the error between $(\boldsymbol{u}, p)$ and the solution to problem (2.13) for $\varepsilon=0$ as performed in [1], Theorem 5.3, in a more general framework and secondly by bounding the error between this solution and $\left(\boldsymbol{u}_{N}^{\varepsilon}, p_{N}^{\varepsilon}\right)$ thanks to [14], Chapter I, Theorem 4.3.

(2) Estimate (2.20) with $\mu=\varepsilon$ is derived firstly by using (2.8), secondly by writing the same formulation as in (2.15)-(2.16) for the solution $\left(\boldsymbol{u}^{\varepsilon}, p^{\varepsilon}\right)$ to problem $(2.7)$, thirdly by bounding successively the errors $\left\|\boldsymbol{u}^{\varepsilon}-\boldsymbol{u}_{N}^{\varepsilon}\right\|_{H^{1}(\Omega)^{d}}$ and $\left\|p^{\varepsilon}-p_{N}^{\varepsilon}\right\|_{L^{2}(\Omega)}$ from this last formulation.

Note that, in practical situations, $\varepsilon$ is most often smaller than $N^{-1}$, so that estimate $(2.20)$ with $\mu=\beta_{N}(\lambda)$ is the best one. Moreover, it only involves the regularity of the solution $(\boldsymbol{u}, p)$.

\section{A POSTERIORI EStimate OF THE PENALty AND DisCRETIZATion ERRORS}

We wish to prove an upper bound for the error between the solutions $(\boldsymbol{u}, p)$ to problem $(2.2)$ and $\left(\boldsymbol{u}_{N}^{\varepsilon}, p_{N}^{\varepsilon}\right)$ to problem (2.13), by a quantity which can be computed explicitly once the discrete solution is known. As is standard in multistep discretizations, the main idea for this is to use the triangle inequalities

$$
\begin{aligned}
\left\|\boldsymbol{u}-\boldsymbol{u}_{N}^{\varepsilon}\right\|_{H^{1}(\Omega)^{d}} & \leq\left\|\boldsymbol{u}-\boldsymbol{u}^{\varepsilon}\right\|_{H^{1}(\Omega)^{d}}+\left\|\boldsymbol{u}^{\varepsilon}-\boldsymbol{u}_{N}^{\varepsilon}\right\|_{H^{1}(\Omega)^{d}} \\
\left\|p-p_{N}^{\varepsilon}\right\|_{L^{2}(\Omega)} & \leq\left\|p-p^{\varepsilon}\right\|_{L^{2}(\Omega)}+\left\|p^{\varepsilon}-p_{N}^{\varepsilon}\right\|_{L^{2}(\Omega)}
\end{aligned}
$$

and to evaluate separately the errors issued from the penalization and the discretization.

Indeed, when subtracting problem (2.7) from problem (2.2), we obtain the following system of residual equations

$$
\begin{array}{ll}
\forall \boldsymbol{v} \in H_{0}^{1}(\Omega)^{d}, & a\left(\boldsymbol{u}-\boldsymbol{u}^{\varepsilon}, \boldsymbol{v}\right)+b\left(\boldsymbol{v}, p-p^{\varepsilon}\right)=0, \\
\forall q \in L_{0}^{2}(\Omega), & b\left(\boldsymbol{u}-\boldsymbol{u}^{\varepsilon}, q\right)=-\varepsilon \int_{\Omega} p^{\varepsilon}(\boldsymbol{x}) q(\boldsymbol{x}) \mathrm{d} \boldsymbol{x} .
\end{array}
$$


Thus, standard arguments (see [14], Chap. I, Cor. 4.1), combined with the ellipticity property (2.4) and the inf-sup condition (2.5), yield the bound

$$
\left\|\boldsymbol{u}-\boldsymbol{u}^{\varepsilon}\right\|_{H^{1}(\Omega)^{d}}+\left\|p-p^{\varepsilon}\right\|_{L^{2}(\Omega)} \leq c \varepsilon\left\|p^{\varepsilon}\right\|_{L^{2}(\Omega)} .
$$

However, in view of the implementation, we wish to define error indicators which only depend on the discrete solution $\left(\boldsymbol{u}_{N}^{\varepsilon}, p_{N}^{\varepsilon}\right)$. So we introduce the error indicator

$$
\eta^{\varepsilon}=\varepsilon\left\|p_{N}^{\varepsilon}\right\|_{L^{2}(\Omega)} .
$$

Since $\eta^{\varepsilon}$ only depends on the discrete solution, it can be easily computed. We are now in a position to state the first part of our a posteriori estimates.

Theorem 3.1. There exists a constant $c$ independent of $\varepsilon$ and $N$ such that the following error estimate holds between the solutions $(\boldsymbol{u}, p)$ to problem $(2.2)$ and $\left(\boldsymbol{u}^{\varepsilon}, p^{\varepsilon}\right)$ to problem $(2.7)$

$$
\left\|\boldsymbol{u}-\boldsymbol{u}^{\varepsilon}\right\|_{H^{1}(\Omega)^{d}}+\left\|p-p^{\varepsilon}\right\|_{L^{2}(\Omega)} \leq c\left(\eta^{\varepsilon}+\varepsilon\left\|p^{\varepsilon}-p_{N}^{\varepsilon}\right\|_{L^{2}(\Omega)}\right) .
$$

The following bound holds for the indicator $\eta^{\varepsilon}$ defined in (3.4)

$$
\eta^{\varepsilon} \leq\left|\boldsymbol{u}-\boldsymbol{u}^{\varepsilon}\right|_{H^{1}(\Omega)^{d}}+\varepsilon\left\|p^{\varepsilon}-p_{N}^{\varepsilon}\right\|_{L^{2}(\Omega)} .
$$

Proof. Estimate (3.5) follows from (3.3) and a triangle inequality. On the other hand, when taking $q$ equal to $p^{\varepsilon}$ in the second line of (3.2) and using the formula

$$
\forall \boldsymbol{v} \in H_{0}^{1}(\Omega)^{d}, \quad|\boldsymbol{v}|_{H^{1}(\Omega)^{d}}^{2}=\|\operatorname{div} \boldsymbol{v}\|_{L^{2}(\Omega)}^{2}+\|\operatorname{curl} \boldsymbol{v}\|_{L^{2}(\Omega) \frac{d(d-1)}{2}}^{2},
$$

we derive

$$
\varepsilon\left\|p^{\varepsilon}\right\|_{L^{2}(\Omega)} \leq\left|\boldsymbol{u}-\boldsymbol{u}^{\varepsilon}\right|_{H^{1}(\Omega)^{d}} .
$$

Combining this relation with a further triangle inequality gives (3.6).

To estimate the discretization error, we follow the approach in [8], Section 4, and [9], Section 3.3, combined with the arguments in [4], Section 2. Indeed, let us set, for all $U=(\boldsymbol{u}, p)$ and $V=(\boldsymbol{v}, q)$,

$$
\mathcal{A}_{\varepsilon}(U, V)=a(\boldsymbol{u}, \boldsymbol{v})+b(\boldsymbol{v}, p)+b(\boldsymbol{u}, q)-\varepsilon \int_{\Omega} p(\boldsymbol{x}) q(\boldsymbol{x}) \mathrm{d} \boldsymbol{x} .
$$

The form $\mathcal{A}_{\varepsilon}(\cdot, \cdot)$ is bilinear and continuous on $\mathcal{X}(\Omega) \times \mathcal{X}(\Omega)$, with

$$
\mathcal{X}(\Omega)=H_{0}^{1}(\Omega)^{d} \times L_{0}^{2}(\Omega) .
$$

Moreover, the following inf-sup condition is proved in [9], Lemma 3.5, as a consequence of (2.4) and (2.5) and with obvious definition of the norm $\|\cdot\|_{\mathcal{X}(\Omega)}$ : There exists a constant $\beta_{*}>0$ independent of $\varepsilon$ such that

$$
\forall U \in \mathcal{X}(\Omega), \quad \sup _{V \in \mathcal{X}(\Omega)} \frac{\mathcal{A}_{\varepsilon}(U, V)}{\|V\|_{\mathcal{X}(\Omega)}} \geq \beta_{*}\|U\|_{\mathcal{X}(\Omega)} .
$$

So we are led to evaluate the residual $\mathcal{A}_{\varepsilon}\left(U^{\varepsilon}-U_{N}^{\varepsilon}, V\right)$, with $U^{\varepsilon}=\left(\boldsymbol{u}^{\varepsilon}, p^{\varepsilon}\right)$ and $U_{N}^{\varepsilon}=\left(\boldsymbol{u}_{N}^{\varepsilon}, p_{N}^{\varepsilon}\right)$.

We first observe from problem (2.13) and the exactness property $(2.11)$ that, for any $V_{N-1}=\left(\boldsymbol{v}_{N-1}, 0\right)$ with $\boldsymbol{v}_{N-1}$ in $\mathbb{X}_{N-1}$,

$$
\mathcal{A}_{\varepsilon}\left(U_{N}^{\varepsilon}, V_{N-1}\right)=\int_{\Omega}\left(\mathcal{I}_{N} \boldsymbol{f}\right)(\boldsymbol{x}) \cdot \boldsymbol{v}_{N-1}(\boldsymbol{x}) \mathrm{d} \boldsymbol{x} .
$$


Thus, we derive from (2.7) and the previous line

$$
\mathcal{A}_{\varepsilon}\left(U^{\varepsilon}-U_{N}^{\varepsilon}, V\right)=\mathcal{A}_{\varepsilon}\left(U^{\varepsilon}-U_{N}^{\varepsilon}, V-V_{N-1}\right)+\int_{\Omega}\left(\boldsymbol{f}-\mathcal{I}_{N} \boldsymbol{f}\right)(\boldsymbol{x}) \cdot \boldsymbol{v}_{N-1}(\boldsymbol{x}) \mathrm{d} \boldsymbol{x},
$$

or equivalently

$$
\begin{aligned}
\mathcal{A}_{\varepsilon}\left(U^{\varepsilon}-U_{N}^{\varepsilon}, V\right)= & \int_{\Omega}\left(\mathcal{I}_{N} \boldsymbol{f}\right)(\boldsymbol{x}) \cdot\left(\boldsymbol{v}-\boldsymbol{v}_{N-1}\right)(\boldsymbol{x}) \mathrm{d} \boldsymbol{x}-\mathcal{A}_{\varepsilon}\left(U_{N}^{\varepsilon}, V-V_{N-1}\right) \\
& +\int_{\Omega}\left(\boldsymbol{f}-\mathcal{I}_{N} \boldsymbol{f}\right)(\boldsymbol{x}) \cdot \boldsymbol{v}(\boldsymbol{x}) \mathrm{d} \boldsymbol{x}
\end{aligned}
$$

Integrating by parts on each $\Omega_{k}$, we can also write

$$
\begin{aligned}
\int_{\Omega}\left(\mathcal{I}_{N} \boldsymbol{f}\right)(\boldsymbol{x}) \cdot\left(\boldsymbol{v}-\boldsymbol{v}_{N-1}\right)(\boldsymbol{x}) \mathrm{d} \boldsymbol{x}-\mathcal{A}_{\varepsilon}\left(U_{N}^{\varepsilon}, V-V_{N-1}\right) & \\
=\sum_{k=1}^{K}\left(\int_{\Omega_{k}}\left(\mathcal{I}_{N} \boldsymbol{f}+\nu \Delta \boldsymbol{u}_{N}^{\varepsilon}-\operatorname{grad} p_{N}^{\varepsilon}\right)(\boldsymbol{x}) \cdot\left(\boldsymbol{v}-\boldsymbol{v}_{N-1}\right)(\boldsymbol{x}) \mathrm{d} \boldsymbol{x}\right. & -\int_{\partial \Omega_{k}}\left(\nu \partial_{n} \boldsymbol{u}_{N}^{\varepsilon}-p_{N}^{\varepsilon} \boldsymbol{n}\right)(\boldsymbol{\tau}) \cdot\left(\boldsymbol{v}-\boldsymbol{v}_{N-1}\right)(\boldsymbol{\tau}) \mathrm{d} \boldsymbol{\tau} \\
& \left.+\int_{\Omega_{k}}\left(\operatorname{div} \boldsymbol{u}_{N}^{\varepsilon}\right)(\boldsymbol{x}) q(\boldsymbol{x}) \mathrm{d} \boldsymbol{x}+\varepsilon \int_{\Omega_{k}} p_{N}^{\varepsilon}(\boldsymbol{x}) q(\boldsymbol{x}) \mathrm{d} \boldsymbol{x}\right) .
\end{aligned}
$$

To go further, we need some notation.

Notation 3.2. For each $k$, let $\Gamma_{k \ell}, 1 \leq \ell \leq L(k)$, be the edges $(d=2)$ or faces $(d=3)$ of $\Omega_{k}$ which are not contained in $\partial \Omega$. We denote by $[\cdot]_{k \ell}$ the jump through each $\Gamma_{k \ell}$.

This leads to the following definition of the error indicators: For $1 \leq k \leq K$,

$$
\begin{aligned}
\eta_{k}=N^{-1}\left\|\mathcal{I}_{N} \boldsymbol{f}+\nu \Delta \boldsymbol{u}_{N}^{\varepsilon}-\operatorname{grad} p_{N}^{\varepsilon}\right\|_{L^{2}\left(\Omega_{k}\right)^{d}} & \\
& +\sum_{\ell=1}^{L(k)} N^{-\frac{1}{2}}\left\|\left[\nu \partial_{n} \boldsymbol{u}_{N}^{\varepsilon}-p_{N}^{\varepsilon} \boldsymbol{n}\right]_{k \ell}\right\|_{L^{2}\left(\Gamma_{k \ell}\right)^{d}}+\left\|\operatorname{div} \boldsymbol{u}_{N}^{\varepsilon}\right\|_{L^{2}\left(\Omega_{k}\right)}
\end{aligned}
$$

The following result deals with approximation error estimates which are derived from duality arguments. Let $\Pi_{N}^{1,0}$ denote the orthogonal projection operator from $H_{0}^{1}(\Omega)$ onto $\mathbb{X}_{N}$ for the scalar product associated with the norm $|\cdot|_{H^{1}(\Omega)}$.

Lemma 3.3. The following estimate is derived for any function $v$ in $H_{0}^{1}(\Omega)$

$$
\left\|v-\Pi_{N}^{1,0} v\right\|_{L^{2}(\Omega)} \leq c \rho_{\Omega} N^{-1}\|v\|_{H^{1}(\Omega)},
$$

where $\rho_{\Omega}$ is equal

(i) to 1 in dimension $d=2$ or if $\Omega$ is convex,

(ii) to $N^{\frac{1}{2}}$ in dimension $d=3$ and when $\Omega$ is not convex.

This estimate still holds for any vector field in $H_{0}^{1}(\Omega)^{d}$.

Proof. We have

$$
\left\|v-\Pi_{N}^{1,0} v\right\|_{L^{2}(\Omega)}=\sup _{\chi \in L^{2}(\Omega)} \frac{\int_{\Omega}\left(v-\Pi_{N}^{1,0} v\right)(\boldsymbol{x}) \chi(\boldsymbol{x}) \mathrm{d} \boldsymbol{x}}{\|\chi\|_{L^{2}(\Omega)}} .
$$


For any $\chi$ in $L^{2}(\Omega)$, the problem

$$
-\Delta \varphi=\chi \quad \text { in } \Omega, \quad \varphi=0 \quad \text { on } \partial \Omega,
$$

has a unique solution $\varphi$ in $H_{0}^{1}(\Omega)$. Moreover, we note that

$$
\begin{aligned}
\int_{\Omega}\left(v-\Pi_{N}^{1,0} v\right)(\boldsymbol{x}) \chi(\boldsymbol{x}) \mathrm{d} \boldsymbol{x} & =\int_{\Omega}\left(\operatorname{grad}\left(v-\Pi_{N}^{1,0} v\right)\right)(\boldsymbol{x}) \cdot(\operatorname{grad} \varphi)(\boldsymbol{x}) \mathrm{d} \boldsymbol{x} \\
& =\int_{\Omega}(\operatorname{grad} v)(\boldsymbol{x}) \cdot\left(\operatorname{grad}\left(\varphi-\Pi_{N}^{1,0} \varphi\right)\right)(\boldsymbol{x}) \mathrm{d} \boldsymbol{x}
\end{aligned}
$$

so that

$$
\int_{\Omega}\left(v-\Pi_{N}^{1,0} v\right)(\boldsymbol{x}) \chi(\boldsymbol{x}) \mathrm{d} \boldsymbol{x} \leq\|v\|_{H^{1}(\Omega)}\left\|\varphi-\Pi_{N}^{1,0} \varphi\right\|_{H^{1}(\Omega)} .
$$

Moreover, the following result is easily derived from [10], Lemma VI.2.5, thanks to an interpolation argument, for any real number $s \geq 0$,

$$
\left\|\varphi-\Pi_{N}^{1,0} \varphi\right\|_{H^{1}(\Omega)} \leq c N^{-s}\|\varphi\|_{H^{s+1}(\Omega)} .
$$

To conclude, we recall that the mapping: $\chi \mapsto \varphi$ is continuous from $L^{2}(\Omega)$ into $H^{s+1}(\Omega)$, with $s \geq \frac{1}{2}$ in the general case and $s \geq 1$ when $\Omega$ is convex. In dimension $d=2$ and when $\Omega$ is not convex, we are led to use a more complex argument: $\varphi$ is the sum of a function $\varphi_{r}$ in $H^{2}(\Omega)$ and of a singular function $S$ with support in a neighbourhood of the nonconvex corners of $\Omega$. The approximation properties of this last function are established in [5], Section 3 .

We omit the proof of the next statement since the result is established in [4], Corollary 2.6, in dimension $d=2$, and the arguments can easily be extended to the case of dimension $d=3$.

Lemma 3.4. The following estimate is derived for any function $v$ in $H_{0}^{1}(\Omega)$, for all $\Omega_{k}, 1 \leq k \leq K$,

$$
\left\|v-\Pi_{N}^{1,0} v\right\|_{L^{2}\left(\partial \Omega_{k}\right)} \leq c N^{-\frac{1}{2}}\|v\|_{H^{1}(\Omega)} .
$$

By applying (3.8) with $U$ equal to $U^{\varepsilon}-U_{N}^{\varepsilon}$ and using (3.9) combined with (3.10), Cauchy-Schwarz inequalities and Lemmas 3.3 and 3.4, we derive the final estimate.

Theorem 3.5. There exists a constant $c$ independent of $\varepsilon$ and $N$ such that the following a posteriori error estimate holds between the solutions $\left(\boldsymbol{u}^{\varepsilon}, p^{\varepsilon}\right)$ to problem (2.7) and $\left(\boldsymbol{u}_{N}^{\varepsilon}, p_{N}^{\varepsilon}\right)$ to problem (2.13)

$$
\left\|\boldsymbol{u}^{\varepsilon}-\boldsymbol{u}_{N}^{\varepsilon}\right\|_{H^{1}(\Omega)^{d}}+\left\|p^{\varepsilon}-p_{N}^{\varepsilon}\right\|_{L^{2}(\Omega)} \leq c\left(\eta^{\varepsilon}+\rho_{\Omega}\left(\sum_{k=1}^{K} \eta_{k}^{2}\right)^{\frac{1}{2}}+\left\|\boldsymbol{f}-\mathcal{I}_{N} \boldsymbol{f}\right\|_{L^{2}(\Omega)^{d}}\right),
$$

where $\rho_{\Omega}$ is equal

(i) to 1 in dimension $d=2$ or if $\Omega$ is convex,

(ii) to $N^{\frac{1}{2}}$ in dimension $d=3$ and when $\Omega$ is not convex.

In dimension $d=2$ or when $\Omega$ is convex, estimate (3.15) is fully optimal and leads to an explicit upper bound for the error. The converse estimate (i.e. the upper bound of each $\eta_{k}$ as a function of the error) would likely be not optimal, see [4], Theorem 2.9. We do not present it because we do not intend to perform adaptivity with respect to $N$.

\section{Penalty adaptation And numerical experiments}

This section is devoted to a numerical comparison of the discretizations with and without penalization and also, in the penalization case, with and without optimization of the penalty parameter. So, we first describe the strategy that is used for this optimization. 


\subsection{Adaptation of the penalty parameter}

Assuming that the source term $\boldsymbol{f}$ is smooth, we work with sufficiently large $N$ for the quantity $\left\|\boldsymbol{f}-\mathcal{I}_{N} \boldsymbol{f}\right\|_{L^{2}(\Omega)^{d}}$ which appears in (3.15) to be negligible with respect to the other terms. We fix a real number $\rho, 0<\rho<1$, and an initial value $\varepsilon^{0}$ of $\varepsilon$. Next, we apply iteratively the following process.

Optimization STeP. For a given value $\varepsilon^{m}$ of $\varepsilon$, we compute the solution $\left(\boldsymbol{u}_{N}^{\varepsilon^{m}}, p_{N}^{\varepsilon^{m}}\right)$ to the corresponding problem (2.15)-(2.16), and the associated error indicators $\eta^{\varepsilon^{m}}$ defined in (3.4) and $\eta_{k}$ defined in (3.11). We also set:

$$
\eta_{(N)}=\left(\sum_{k=1}^{K} \eta_{k}^{2}\right)^{\frac{1}{2}}
$$

Next, when

$$
\rho \eta_{(N)} \leq \eta^{\varepsilon^{m}} \leq \frac{1}{\rho} \eta_{(N)}
$$

we stop the process. Otherwise, we take $\varepsilon^{m+1}$ equal to a constant times $\varepsilon^{m} \eta_{(N)} / \eta^{\varepsilon^{m}}$.

The optimization step is iterated until condition (4.2) is satisfied (when possible) or only a limited number of times $M_{\max }$.

Remark 4.1. The algorithm for computing the operator: $\boldsymbol{v}_{N} \mapsto \Pi_{N}\left(\operatorname{div} \boldsymbol{v}_{N}\right)$ plays a key role in the implementation of the penalized discrete problem. So we now describe it, in the case $d=2$ for simplicity. We first note from definition (2.10) that the projection operator $\Pi_{N}$ reduces to local ones: More precisely, if $\Pi_{N}^{k}$ denotes the orthogonal projection operator from $L^{2}\left(\Omega_{k}\right)$ onto $\mathbb{P}_{N-2}\left(\Omega_{k}\right) \cap \mathbb{P}_{\lambda N}\left(\Omega_{k}\right)$, for any $\varphi$ in $L_{0}^{2}(\Omega),\left(\Pi_{N} \varphi\right)_{\mid \Omega_{k}}$ coincides with $\Pi_{N}^{k}\left(\varphi \mid \Omega_{k}\right)$. So, without restriction, we only work on the reference square $\left.\hat{\Omega}=\right]-1,1\left[2\right.$ and we denote by $\widehat{\Pi}_{N}$ the corresponding projection operator. Let $\varphi_{i}$ be the Lagrange polynomials associated with the nodes $\xi_{j}$ : For each $i, 0 \leq i \leq N, \varphi_{i}$ belongs to $\mathbb{P}_{N}(-1,1)$ and satisfies: $\varphi_{i}\left(\xi_{j}\right)=\delta_{i j}$. Any function $\boldsymbol{v}_{N}$ in $\mathbb{P}_{N}(\widehat{\Omega})^{2}$ can be written as

$$
\boldsymbol{v}_{N}(\zeta, \xi)=\sum_{i=0}^{N} \sum_{j=0}^{N} \boldsymbol{v}^{i j} \varphi_{i}(\zeta) \varphi_{j}(\xi),
$$

where each $\boldsymbol{v}^{i j}=\left(v_{1}^{i j}, v_{2}^{i j}\right)$ is equal to $\boldsymbol{v}_{N}\left(\xi_{i}, \xi_{j}\right)$. Thus, we have

$$
\left(\operatorname{div} \boldsymbol{v}_{N}\right)(\zeta, \xi)=\sum_{i=0}^{N} \sum_{j=0}^{N}\left(v_{1}^{i j} \varphi_{i}^{\prime}(\zeta) \varphi_{j}(\xi)+v_{2}^{i j} \varphi_{i}(\zeta) \varphi_{j}^{\prime}(\xi)\right) .
$$

Denoting by $L_{n}, n \geq 0$, the Legendre polynomials, we also have the expansion

$$
\left(\operatorname{div} \boldsymbol{v}_{N}\right)(\zeta, \xi)=\sum_{\ell=0}^{N} \sum_{n=0}^{N} d_{\ell n} L_{\ell}(\zeta) L_{n}(\xi),
$$

so that

$$
\widehat{\Pi}_{N}\left(\operatorname{div} \boldsymbol{v}_{N}\right)(\zeta, \xi)=\left(\operatorname{div} \boldsymbol{v}_{N}\right)(\zeta, \xi)-\sum_{(\ell, n) \in \mathcal{N}} d_{\ell n} L_{\ell}(\zeta) L_{n}(\xi),
$$

where $\mathcal{N}$ stands for the set of pair of indices $(\ell, n), 0 \leq \ell, n \leq N$, such that either $\ell$ or $n$ is larger than $\min \{N-2, \lambda N\}$. It remains to compute the $d_{\ell n}$ as a function of the $\boldsymbol{v}^{i j}$. It follows from (4.4) that

$$
d_{\ell n}=\sum_{i=0}^{N} \sum_{j=0}^{N}\left(v_{1}^{i j} \beta_{\ell}^{i} \alpha_{n}^{j}+v_{2}^{i j} \alpha_{\ell}^{i} \beta_{n}^{j}\right),
$$


with

$$
\alpha_{n}^{j}=\frac{1}{\left\|L_{n}\right\|_{L^{2}(-1,1)}^{2}} \int_{-1}^{1} \varphi_{j}(\zeta) L_{n}(\zeta) \mathrm{d} \zeta, \quad \beta_{n}^{j}=\frac{1}{\left\|L_{n}\right\|_{L^{2}(-1,1)}^{2}} \int_{-1}^{1} \varphi_{j}^{\prime}(\zeta) L_{n}(\zeta) \mathrm{d} \zeta .
$$

From the formulas (see [6], Thm. 3.2 and form. (13.19))

$$
\left\|L_{n}\right\|_{L^{2}(-1,1)}^{2}=\frac{1}{n+\frac{1}{2}}, \quad \sum_{i=0}^{N} L_{N}^{2}\left(\xi_{i}\right) \rho_{i}=\frac{2}{N},
$$

combined with (2.11), we derive

$$
\alpha_{n}^{j}=c(n) L_{n}\left(\xi_{j}\right) \rho_{j} \quad \text { with } \quad c(n)= \begin{cases}n+\frac{1}{2} & \text { if } 0 \leq n<N \\ \frac{N}{2} & \text { if } n=N .\end{cases}
$$

Evaluating the $\beta_{n}^{j}$ requires a further integration by parts:

$$
\beta_{n}^{j}=\left(n+\frac{1}{2}\right)\left(-L_{n}^{\prime}\left(\xi_{j}\right) \rho_{j}+\delta_{j N}-(-1)^{n} \delta_{j 0}\right) .
$$

\subsection{First computations}

We conclude with several types of numerical experiments, in dimension $d=2$. We first take the source term $\boldsymbol{f}$ equal to zero and the boundary condition $\boldsymbol{u}=\mathbf{0}$ replaced by

$$
\boldsymbol{u}=\boldsymbol{g} \quad \text { on } \partial \Omega,
$$

for a continuous function $\boldsymbol{g}$ in $H^{\frac{1}{2}}(\partial \Omega)^{2}$, satisfying

$$
\int_{\partial \Omega}(\boldsymbol{g} \cdot \boldsymbol{n})(\tau) \mathrm{d} \tau=0
$$

The corresponding discrete condition reads

$$
\boldsymbol{u}_{N}^{\varepsilon}=\boldsymbol{g}_{N} \quad \text { on } \partial \Omega,
$$

where $\boldsymbol{g}_{N}$ is the interpolate of $\boldsymbol{g}$ at all nodes $F_{k}\left(\xi_{i}, \xi_{j}\right)$ which belong to $\partial \Omega$, with values in the trace space of $\mathbb{X}_{N}$. Note that the previous analysis easily extends to this new situation.

The numerical experiments deal either with the square $\Omega=]-1,1\left[{ }^{2}\right.$ without domain decomposition or with the $L$-shaped domain $\Omega=]-1,1\left[{ }^{2} \backslash\left[0,1\left[^{2}\right.\right.\right.$ divided into three equal squares in an obvious way. These domains and the corresponding Gauss-Lobatto grids for $N=20$ are illustrated in Figure 1.

We take the viscosity $\nu$ equal to $10^{-2}$ while for $\boldsymbol{g}$ we make the following choice:

- When $\Omega$ is the square, we consider a regularized driven cavity problem

$$
\boldsymbol{g}(x,-1)=\boldsymbol{g}(-1, y)=\boldsymbol{g}(1, y)=\mathbf{0}, \quad \boldsymbol{g}(x, 1)=\left(\begin{array}{c}
\left(1-x^{2}\right)^{\frac{5}{2}} \\
0
\end{array}\right), \quad-1 \leq x, y \leq 1
$$

- When $\Omega$ is the $L$-shaped domain, we consider a Poiseuille type flow

$$
\begin{aligned}
\boldsymbol{g}(-1, y) & =\left(\begin{array}{c}
1-y^{2} \\
0
\end{array}\right), \quad-1 \leq y \leq 1 \\
\boldsymbol{g}(1, y) & =\left(\begin{array}{c}
-8 y(1+y) \\
0
\end{array}\right), \quad-1 \leq y \leq 0 \\
\boldsymbol{g} & =\mathbf{0} \quad \text { elsewhere. }
\end{aligned}
$$




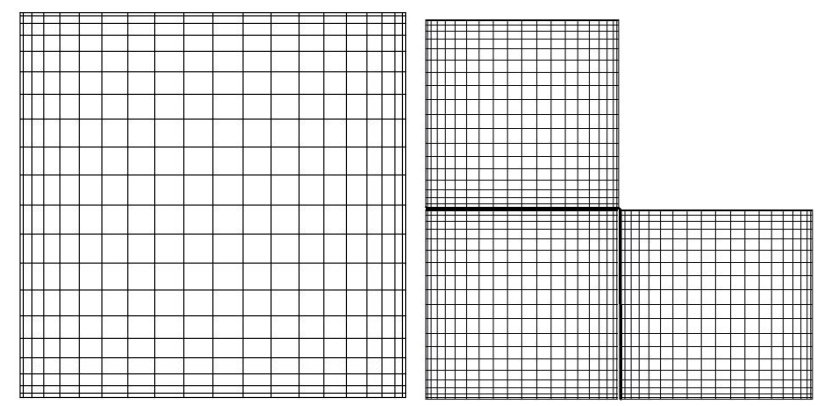

Figure 1. The computation domains and examples of Gauss-Lobatto grids.
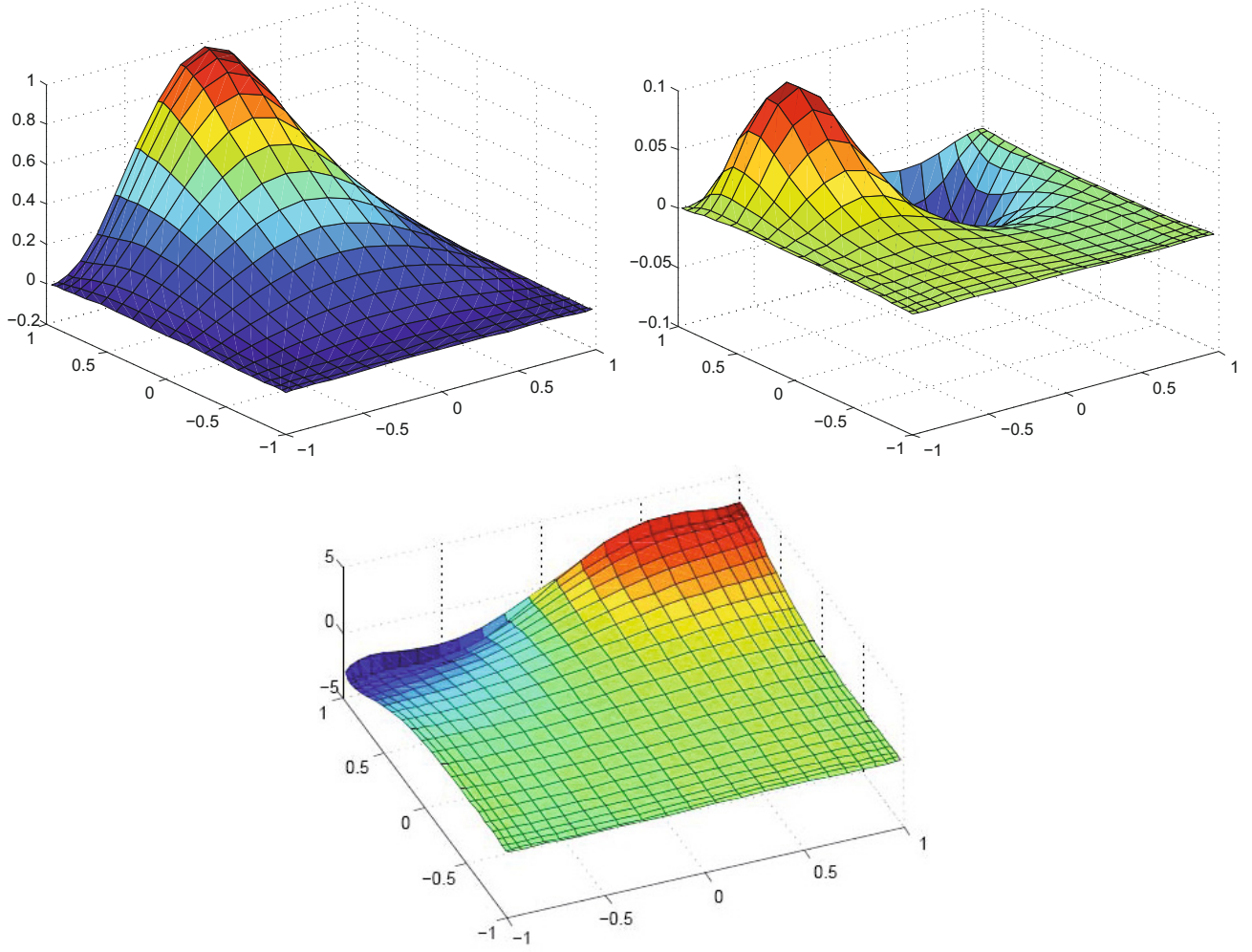

Figure 2. The two components of the velocity and the pressure for the boundary condition in (4.13).

Figures 2 and 3 present from top to bottom the curves of isovalues of the two components of the velocity and of the pressure for these two problems, obtained with $N=30$ and $\varepsilon=10^{-5}$.

\subsection{Optimization of the penalty parameter}

We now work on the $L$-shaped domain, again with $\nu=10^{-2}$. We consider the solution $(\boldsymbol{u}=\operatorname{curl} \psi, p)$ given by

$$
\psi(x, y)=\left(1-x^{2}\right)^{\frac{5}{2}}\left(1-y^{2}\right)^{\frac{5}{2}} \sin (\pi x) \sin (\pi y), \quad p(x, y)=x y+\frac{1}{12}
$$



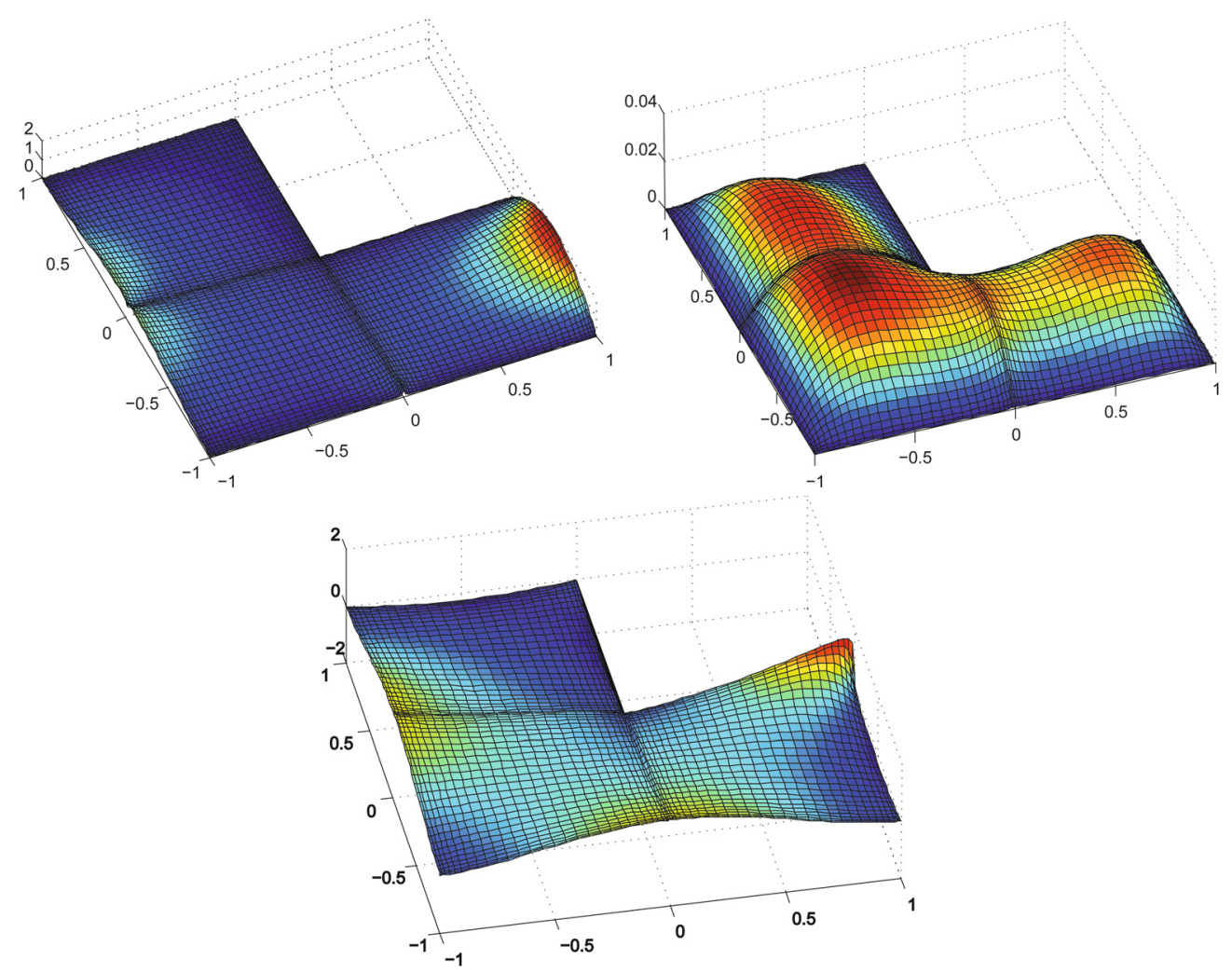

Figure 3. The two components of the velocity and the pressure for the boundary condition in (4.14).

We first study the influence of $\varepsilon$ and $N$ on the indicators $\eta^{\varepsilon}$ and $\eta_{(N)}$. For $N$ fixed equal to 30 and $\varepsilon$ varying between $10^{-1}$ and $10^{-5}$, Figure 4 presents the error $\left\|\boldsymbol{u}-\boldsymbol{u}_{N}^{\varepsilon}\right\|_{H^{1}(\Omega)^{d}}$ (plain red line), the error indicators $\eta^{\varepsilon}$ (dashed dotted blue line) and $\eta_{(N)}$ (dashed black line). It can thus be checked that the $\eta_{(N)}$ are fully independent of $\varepsilon$ (we refer to [9], Sect. 5, for similar results in the finite element case). Moreover the error and $\eta^{\varepsilon}$ decrease with exactly the same slope until the discretization error becomes larger than the penalization error.

Similarly, for $\varepsilon$ fixed equal to $10^{-5}$ and $N$ varying between 10 and 35, Figure 5 represents the full error $\left\|\boldsymbol{u}-\boldsymbol{u}_{N}^{\varepsilon}\right\|_{H^{1}(\Omega)^{d}}$ (plain red line), the error indicators $\eta^{\varepsilon}$ (dashed black line) and $\eta_{(N)}$ (dashed dotted blue line). Here, the $\eta^{\varepsilon}$ are completely independent of $N$.

We still work with the solution $(\boldsymbol{u}, p)$ defined from (4.15). We apply the optimization strategy for $\varepsilon$ described in Section 4.1, with $\rho=0.8$ and $M_{\max }=5$. Table 1 presents for five values of $N$ the different values of the optimized $\varepsilon$ (where "optimized" means that (4.2) holds or that $M_{\max }=5$ iterations have been performed), denoted by $\varepsilon_{\mathrm{opt}}$. It can be observed that $\varepsilon_{\mathrm{opt}}$ quickly decreases when $N$ increases, which seems in good coherence with the previous analysis. Indeed, since the solution $(\boldsymbol{u}, p)$ is smooth, the error $\left\|\boldsymbol{u}^{\varepsilon}-\boldsymbol{u}_{N}^{\varepsilon}\right\|_{H^{1}(\Omega)^{d}}$ also quickly decreases when $N$ increases.

\subsection{Discretization with and without penalty}

In order to check the efficiency of the penalty algorithm, we first compare three algorithms: The Uzawa method (which is another well-known algorithm for uncoupling the two unknowns, see [15], Sect. 3.1, for instance) combined with Conjugate Gradient iterations, the penalty method with $\varepsilon=10^{-5}$ and the penalty method with optimized $\varepsilon$. Table 2 presents the CPU time needed on the computer based on Intel Pentium (4 CPU $3.06 \mathrm{GHz}$ ) to invert the final system resulting from the three methods. 


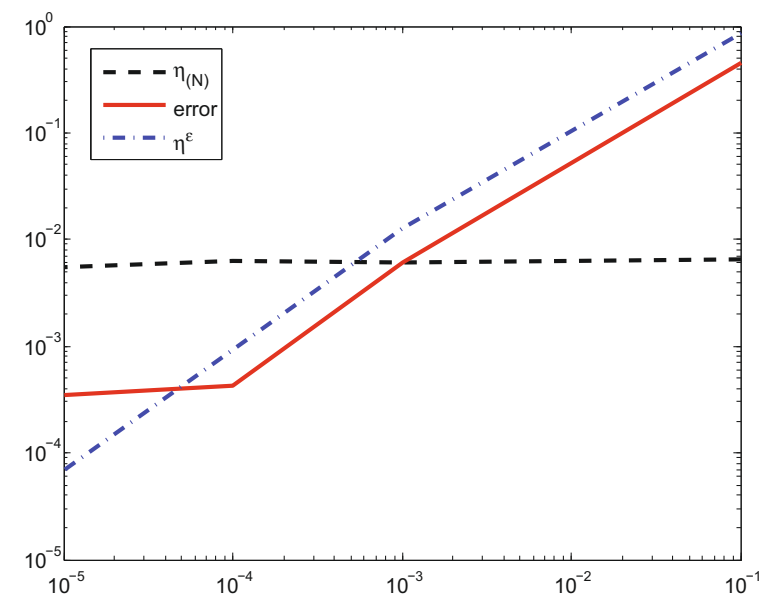

Figure 4. The error and the indicators $\eta^{\varepsilon}$ and $\eta_{(N)}$ for a fixed $N$.

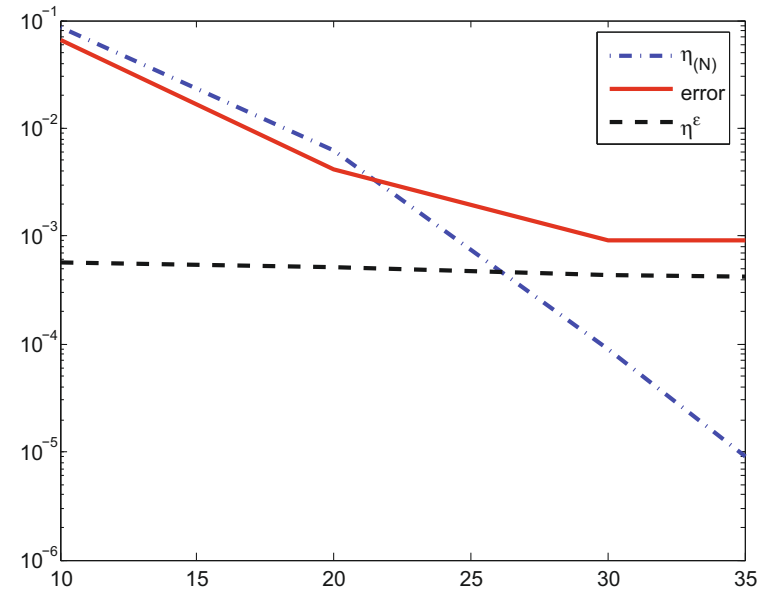

Figure 5. The error and the indicators $\eta^{\varepsilon}$ and $\eta_{(N)}$ for a fixed $\varepsilon$.

TABLE 1 . Values of $\varepsilon_{\mathrm{opt}}$ as a function of $N$.

\begin{tabular}{|c|c|c|c|c|c|}
\hline$N$ & 5 & 10 & 15 & 20 & 30 \\
\hline$\varepsilon_{\mathrm{opt}}$ & 0.0160 & 0.0088 & 0.0063 & 0.0016 & 0.0009 \\
\hline
\end{tabular}

TABle 2. Comparison of the CPU times for the three algorithms.

\begin{tabular}{|c|c|c|c|c|c|}
\hline$N$ & 5 & 10 & 15 & 20 & 30 \\
\hline Uzawa & 3.9211 & 6.0961 & 45.722 & 83.184 & 131.53 \\
$\varepsilon=10^{-5}$ & 3.1607 & 5.9128 & 25.201 & 70.901 & 99.432 \\
$\varepsilon_{\text {opt }}$ & 1.9131 & 4.5512 & 11.294 & 40.706 & 98.841 \\
\hline
\end{tabular}



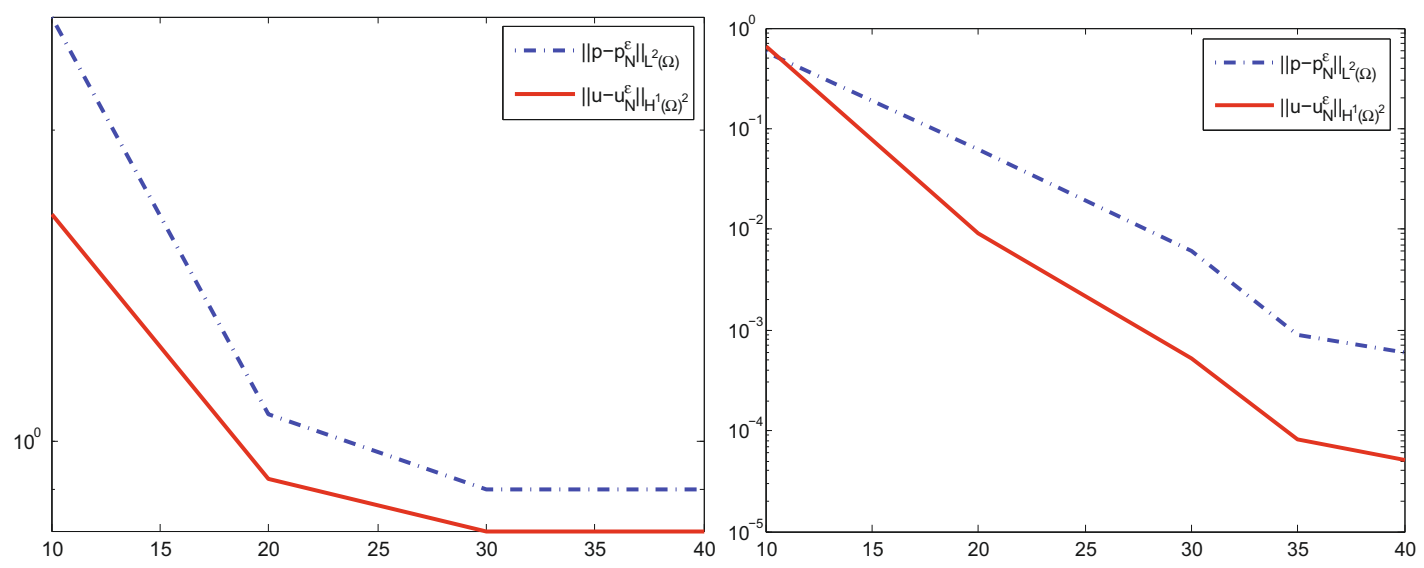

FiguRE 6 . The errors for the space $\mathbb{M}_{N}^{+}$(Uzawa and penalization algorithms).

As well-known, the penalty method with any reasonable choice of $\varepsilon$ is less expensive than the Uzawa algorithm. Moreover, optimizing $\varepsilon$ allows us to reduce the computation cost at least for low values of $N$ (since $\varepsilon_{\text {opt }}$ becomes closer to $10^{-5}$ when $N$ increases). Note also that the cost of the optimization process is negligible with respect to the final computation.

\subsection{About the choice of the pressure space}

We compare the convergence for three choices of discrete pressure spaces:

(i) The space

$$
\mathbb{M}_{N}^{+}=\left\{q_{N} \in L_{0}^{2}(\Omega) ; q_{N \mid \Omega_{k}} \in \mathbb{P}_{N-1}\left(\Omega_{k}\right), 1 \leq k \leq K\right\} .
$$

(ii) The space $\mathbb{M}_{N}$ defined in (2.10) with $\lambda=1$.

(iii) The space $\mathbb{M}_{N}$ defined in (2.10) with $\lambda=0.9$.

It is well-known ([6], Sect. 24) that the space $\mathbb{M}_{N}^{+}$contains at least one spurious mode for the pressure, so that the non-penalized discrete problem is a priori not well-posed. In contrast, the same arguments as for Proposition 2.2 yield that the penalized discrete problem has a unique solution (but no convergence can be established).

We again work with the square $\Omega=]-1,1[2$, for the exact solution $(\boldsymbol{u}=\operatorname{curl} \psi, p)$ now given by

$$
\psi(x, y)=\left(1-x^{2}\right)^{\frac{5}{2}}\left(1-y^{2}\right)^{\frac{5}{2}}, \quad p(x, y)=x y
$$

We first deal with the space $\mathbb{M}_{N}^{+}$defined in (4.16). For $N$ varying from 5 to 40, Figure 6 presents the curves for the errors $\left\|\boldsymbol{u}-\boldsymbol{u}_{N}^{\varepsilon}\right\|_{H^{1}(\Omega)^{2}}$ (plain red line) and $\left\|p-p_{N}^{\varepsilon}\right\|_{L^{2}(\Omega)}$ (dashed dotted blue line), obtained either with the Uzawa method (on the left) and the penalization method with $\varepsilon=10^{-5}$ (on the right). There is no convergence for the Uzawa algorithm and the convergence for the penalization algorithm is of low order.

Figure 7 presents the same curves for the errors, now obtained with the penalization method with $\varepsilon=10^{-5}$ for the spaces $\mathbb{M}_{N}$ with $\lambda=1$ (on the left) and $\lambda=0.9$ (on the right). In both cases, the convergence is of spectral type, i.e., of order only limited by the regularity of the exact solution as appears in (2.20). The convergence order also slightly increases when $\lambda=0.9$, in good agreement with the fact that the parameter $\mu$ in (2.20) is equal to 1 in this case.

To conclude, we present in the following Table 3 the values of $\eta_{(N)}$ obtained with $\varepsilon$ fixed equal to $10^{-5}$ and of $\varepsilon_{\text {opt }}$ (computed by the optimization strategy described in Section 4.1 , with $\varepsilon^{0}=1, \rho=0.8$ and $M_{\max }=5$ ) for the three choices of spaces of pressure. When compared with the previous figures, this table indicates that the $\eta_{(N)}$ provide a good representation of the discretization error. But, when starting with $\varepsilon^{0}=1, M_{\max }=5$ 

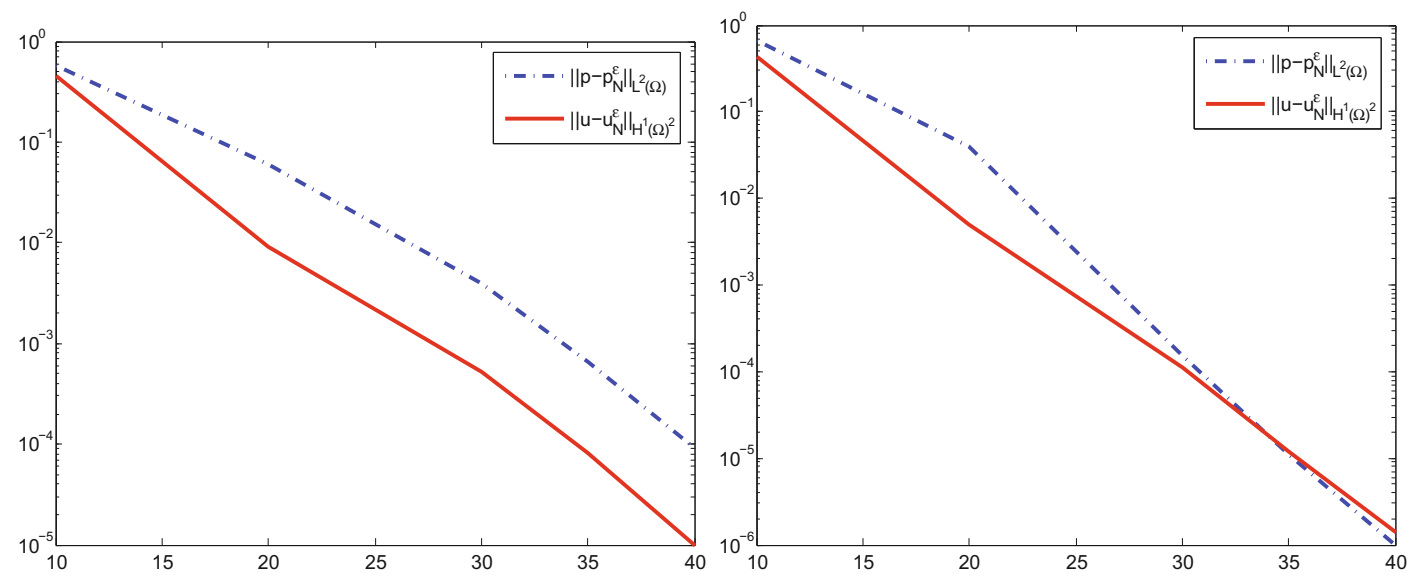

FiguRE 7. Velocity and pressure errors for two different choices of spaces $\mathbb{M}_{N}$.

TABLE 3. Comparison of $\eta_{(N)}$ and of $\varepsilon_{\mathrm{opt}}$ for the different spaces of pressures.

\begin{tabular}{|c|c|c|c|c|c|c|}
\hline & $N$ & 5 & 10 & 20 & 30 & 40 \\
\hline Choice (i) & $\eta_{(N)}$ & 0.857 & 0.620 & $0.91 \times 10^{-2}$ & $0.13 \times 10^{-3}$ & $0.80 \times 10^{-4}$ \\
& $\varepsilon_{\text {opt }}$ & 0.521 & 0.585 & $0.75 \times 10^{-1}$ & $0.29 \times 10^{-1}$ & $0.85 \times 10^{-2}$ \\
\hline Choice (ii) & $\eta_{(N)}$ & $0.18 \times 10^{-1}$ & $0.93 \times 10^{-3}$ & $0.81 \times 10^{-4}$ & $0.13 \times 10^{-5}$ & $10^{-8}$ \\
& $\varepsilon_{\text {opt }}$ & $0.98 \times 10^{-2}$ & $0.72 \times 10^{-2}$ & $0.15 \times 10^{-2}$ & $0.87 \times 10^{-3}$ & $0.24 \times 10^{-3}$ \\
\hline Choice (iii) & $\eta_{(N)}$ & $0.18 \times 10^{-1}$ & $0.93 \times 10^{-3}$ & $0.81 \times 10^{-4}$ & $0.13 \times 10^{-5}$ & $10^{-8}$ \\
& $\varepsilon_{\text {opt }}$ & $0.98 \times 10^{-2}$ & $0.72 \times 10^{-2}$ & $0.15 \times 10^{-2}$ & $0.25 \times 10^{-3}$ & $10^{-4}$ \\
\hline
\end{tabular}

iterations do not seem sufficient to optimize $\varepsilon$, i.e., to obtain that the penalization and discretization errors are of the same order. Further numerical experiments are under consideration.

Acknowledgements. The authors are very grateful toward Frédéric Hecht for his clever comments on the numerical computations and the way of improving them.

\section{REFERENCES}

[1] F. Ben Belgacem, C. Bernardi, N. Chorfi and Y. Maday, Inf-sup conditions for the mortar spectral element discretization of the Stokes problem. Numer. Math. 85 (2000) 257-281.

[2] M. Bercovier, Régularisation duale des problèmes variationnels mixtes : application aux éléments finis mixtes et extension à quelques problèmes non linéaires. Thèse de Doctorat d'État, Université de Rouen, France (1976).

[3] M. Bercovier, Perturbation of mixed variational problems. Application to mixed finite element methods. RAIRO Anal. Numér. 12 (1978) 211-236.

[4] C. Bernardi, Indicateurs d'erreur en $h-N$ version des éléments spectraux. RAIRO Modél. Math. Anal. Numér. 30 (1996) 1-38.

[5] C. Bernardi and Y. Maday, Polynomial approximation of some singular functions. Appl. Anal. 42 (1991) 1-32.

[6] C. Bernardi and Y. Maday, Spectral Methods, in Handbook of Numerical Analysis V, P.G. Ciarlet and J.-L. Lions Eds., North-Holland (1997) 209-485.

[7] C. Bernardi and Y. Maday, Uniform inf-sup conditions for the spectral discretization of the Stokes problem. Math. Mod. Meth. Appl. Sci. 9 (1999) 395-414.

[8] C. Bernardi, B. Métivet and R. Verfürth, Analyse numérique d'indicateurs d'erreur, in Maillage et adaptation, P.-L. George Ed., Hermès (2001) 251-278.

[9] C. Bernardi, V. Girault and F. Hecht, A posteriori analysis of a penalty method and application to the Stokes problem. Math. Mod. Meth. Appl. Sci. 13 (2003) 1599-1628. 
[10] C. Bernardi, Y. Maday and F. Rapetti, Discrétisations variationnelles de problèmes aux limites elliptiques, Mathématiques \& Applications 45. Springer-Verlag (2004).

[11] G.F. Carey and R. Krishnan, Penalty approximation of Stokes flow. Comput. Meth. Appl. Mech. Eng. 35 (1982) 169-206.

[12] G.F. Carey and R. Krishnan, Penalty finite element method for the Navier-Stokes equations. Comput. Meth. Appl. Mech. Eng. 42 (1984) 183-224.

[13] G.F. Carey and R. Krishnan, Convergence of iterative methods in penalty finite element approximation of the Navier-Stokes equations. Comput. Meth. Appl. Mech. Eng. 60 (1987) 1-29.

[14] V. Girault and P.-A. Raviart, Finite Element Methods for Navier-Stokes Equations, Theory and Algorithms. Springer-Verlag (1986).

[15] Y. Maday, D. Meiron, A.T. Patera and E.M. Rønquist, Analysis of iterative methods for the steady and unsteady Stokes problem: Application to spectral element discretizations. SIAM J. Sci. Comput. 14 (1993) 310-337.

[16] D.S. Malkus and E.T. Olsen, Incompressible finite elements which fail the discrete LBB condition, in Penalty-Finite Element Methods in Mechanics, Phoenix, Am. Soc. Mech. Eng., New York (1982) 33-50. 